\title{
Comparative Analysis of Correlation Shift Discriminators
}

\author{
V. N. Kruglov \\ Institute of Radio Engineering and Information Technologies, Ural Federal University, \\ ul. Mira 19, Yekaterinburg, 620002 Russia \\ e-mail:v.krouglov@mail.ru
}

\begin{abstract}
A comparative analysis of correlation shift discriminators is carried out regarding the stabilization accuracy of their discriminator curve (DC). A modification of the classical correlation discriminator is proposed that provides higher accuracy of estimating a shift between signals. It is shown that the modified discriminator is free of "edge effects."
\end{abstract}

Keywords: correlation-extremal tracking system, superimposition of images, correlation discriminator, discriminator curve, edge effect.

DOI: $10.1134 / \mathrm{S} 1054661813030085$

\section{INTRODUCTION}

Correlation discriminators (CDs) of shifts serve as measurement units in optoelectronic tracking systems (OETSs) equipped with machine vision, which yield a solution to navigation problems of keeping a given direction or determining the shift and velocity of a tracked object within the coverage zone by comparing the observed and reference images. In [1], the principles of synthesis of searchless CD algorithms have been generalized. The main advantages of such algorithms over multistep global extremum search procedures are as follows:

-much lower computational cost, because the algorithms dispense with searching for the extremum of a two-dimensional similarity function;

- the possibility of stepless measurement of shifts of an image on a continuous scale of values irrespective of its spatial sampling increment, owing to which the shifts can be tracked with an accuracy that is not directly related to the resolution of images.

A CD of a one-dimensional shift of images serves as a measurement unit that synchronously receives current and reference video frames at two inputs and puts out an estimate for the shifts between the input signals after the end of each subsequent pair of signals. A twodimensional $\mathrm{CD}$ is formed by partial discriminators each of which estimates one component of the additive shift, either in the direction of rows or in the direction of columns.

The most comprehensive account of the problem of estimating the mutual displacement of two images is given in [1-8]. In [1], the authors present the design principles of correlation-extremal algorithms that form the basis of OETSs. In [6-8], the elements of correlation theory are presented, which were devel- oped and complemented as applied to CDs of images. These papers also describe a method for constructing CDs of images on the basis of a weighted orthocorrelation characteristic (OCC) of signals.

The principle of synthesizing searchless CDs based on weighted OCCs of signals (OCC CDs) is as follows. In general, the algorithm for calculating the "orthocorrelation coefficient" of a finite signal describes the procedure of obtaining a normalized linear estimate for the shift between its current and reference samples and proceeds as follows. Let us identify finite signals $f_{\mathrm{r}}(t)$ and $f_{\mathrm{c}}(t)=f_{\mathrm{r}}(t-\tau)$ with vectors $x_{\mathrm{r}}$ and $x_{\mathrm{c}}$ in the basis of two mutually orthogonal normalized finite functions $C(t)$ and $S(t)$ that are even and odd with respect to the midpoint of an interval $T$, respectively; they are such that

$$
\begin{gathered}
C(t)=C(-t), \quad S(t)=-S(-t), \quad \forall t \subset\{ \pm T / 2\}, \\
C(t)=0, \quad S(t)=0, \quad \forall t \not \subset\{ \pm T / 2\}, \\
(S(t), C(t))=0 .
\end{gathered}
$$

Then, in the presence or absence of a shift $\tau$, we obtain the representations

$$
f_{\mathrm{r}}(t)=x_{\mathrm{r}}=\left[a_{\mathrm{r}}, b_{\mathrm{r}}\right]^{T} \text { and } f_{\mathrm{c}}(t)=x_{\mathrm{c}}=\left[a_{\mathrm{c}}, b_{\mathrm{c}}\right]^{T} \text {, }
$$

where the expansion coefficients of the vectors are given by the scalar products

$$
\begin{array}{ll}
a_{\mathrm{r}}=\left(C(t), f_{\mathrm{r}}(t)\right), & b_{\mathrm{r}}=\left(S(t), f_{\mathrm{r}}(t)\right), \\
a_{\mathrm{c}}=\left(C(t), f_{\mathrm{c}}(t)\right), & b_{\mathrm{c}}=\left(S(t), f_{\mathrm{c}}(t)\right),
\end{array}
$$

as the results of nonstationary linear filtration of the compared processes. Orthogonalizing the vector $\mathbf{x}_{\mathrm{r}}$ and multiplying it by the rotation matrix

$$
A=\left(\begin{array}{cc}
0 & -1 \\
1 & 0
\end{array}\right),
$$




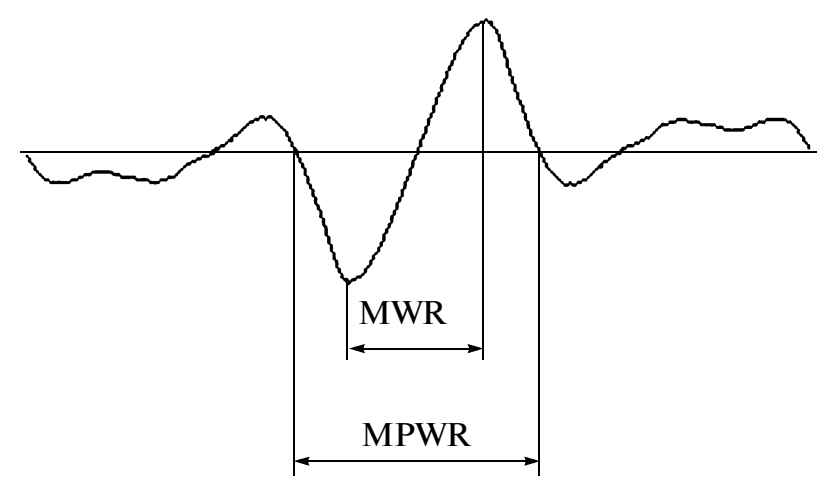

Fig. 1. Maximum permissible working range (MPWR) and monotonic working range (MWR) of discriminator curve.

we obtain the orthocorrelation function of the original (reference) and shifted (current) signals in the form [8]

$$
R(\tau)=a_{\mathrm{r}} b_{\mathrm{c}}-a_{\mathrm{c}} b_{\mathrm{r}} .
$$

As the basis functions $C(t)$ and $S(t)$, Gapon [7], in particular, suggests using segments of harmonic functions that contain an integer number $l$ of periods:

$$
C(t)=\cos (\omega t) \text { and } S(t)=\sin (\omega t), \quad \omega=\frac{2 \pi l}{L} .
$$

One can distinguish two main stages in the operation of OETSs [3-5]:

—automatic target lock-in;

— target autotracking.

In the design of OETSs for autotracking (AT) of images, special attention is paid to the transition stage when the system enters the tracking mode. At this stage, the system automatically eliminates rough errors of preliminary targeting and starts to keep a given direction with small errors. Using the terminology adopted in [6] and presented in Fig. 1, we can formulate the results of these studies as follows.

At the first stage, the CD should have the maximum permissible working range (MPWR) of maximum width, and, at the second stage, the CD should provide maximum linearization of the monotonic working range (MWR). In addition, it is desirable to have an MWR that would provide an estimate for the mutual displacement of images with a pixel, or even a subpixel, accuracy for a two-dimensional signal.

In [6], Firsov analyzed various basis functions and normalization techniques for the steepness of the slope of a partial discriminator curve (DC). In [7], the attainable accuracy of superimposition of images was estimated by weighted orthocorrelation functions. In [2], the authors analyzed the influence of edge effects on the zero drift and the shape of a DC. The results of these investigations can be summarized as follows:
- the most suitable basis functions that guarantee the maximum width of the MPWR are functions of the form

$$
\begin{gathered}
C(t)=\cos (\omega t)+\cos (2 \omega t) \\
\text { and } S(t)=\sin (\omega t)+\sin (2 \omega t) ;
\end{gathered}
$$

- among various algorithms for the normalization of the steepness of a DC, the best results are shown by the method of normalization at zero, which consists in direct measurement of the slope of the DC using test shifts (earlier, this result was obtained independently in [9]);

- the maximum attainable accuracy of superimposition of two-dimensional signals is 0.07 pixels for an image size of $100 \times 100$ pixels;

- the influence of edge effects on the output characteristics increases in proportion to the shift.

In addition, it has been pointed out in [7] that, according to the author, there is hardly any universal normalization method that would give an optimal result for all possible cases, and one should choose a normalization method individually for given sets of basis functions and tracking loop parameters.

To eliminate the influence of edge effects when the signals are shifted with respect to each other within the MWR, to increase the accuracy of their superimposition, and to guarantee stable steepness of the DC for a sufficiently wide MPWR, we propose a new method for constructing CDs.

\section{MAIN PART}

According to the theory of differential correlationextremal systems [1], the DC of a tracking system is the derivative of the autocorrelation function of the reference signal $f_{\mathrm{r}}(t)$ under processing:

$$
D(\tau)=K^{\prime}(\tau)=\sum_{t=0}^{L-1} f_{\mathrm{r}}^{\prime}(t) f_{\mathrm{r}}(t+\tau) .
$$

The control signal generated by the tracking system is calculated as follows:

$$
D\left(t_{0}\right)=\sum_{t=0}^{L-1} f_{\mathrm{r}}^{\prime}(t) f_{\mathrm{c}}(t),
$$

where $f_{\mathrm{c}}(t)$ is a current signal related to $f_{\mathrm{r}}(t)$ by the formula $f_{\mathrm{c}}(t)=f_{\mathrm{r}}\left(t-t_{0}\right)$.

The derivative of the reference finite discrete signal is approximated by the first difference:

$$
f_{\mathrm{r}}^{\prime}(t)=f_{\mathrm{r}}(t-1)-f_{\mathrm{r}}(t+1) .
$$

Therefore, formula (2) reduces to

$$
D\left(t_{0}\right)=\sum_{t=0}^{L-1}\left[f_{\mathrm{r}}(t-1)-f_{\mathrm{r}}(t+1)\right] f_{\mathrm{c}}(t) .
$$


Here $f_{\mathrm{r}}(-1)$ and $f_{\mathrm{r}}(L)$ are taken to be zero. Opening the brackets in (4) and substituting $f_{\mathrm{r}}(t)$ for $f_{\mathrm{c}}(t)$ and $\tau$ for $t_{0}$, we obtain

$$
\begin{gathered}
D(\tau)=\sum_{t=0}^{L-1}\left[f_{\mathrm{r}}(t-1)-f_{\mathrm{r}}(t+1)\right] f_{\mathrm{c}}(t) \\
=\sum_{t=0}^{L-1} f_{\mathrm{r}}(t-1) f_{\mathrm{r}}(t-\tau)-\sum_{t=0}^{L-1} f_{\mathrm{r}}(t+1) f_{\mathrm{r}}(t-\tau) \\
=K(\tau-1)-K(\tau+1) .
\end{gathered}
$$

Formula (5) shows that $D(\tau)$ represents the difference of two autocorrelation functions $K(\tau-1)$ and $K(\tau+1)$ of the reference signal $f_{\mathrm{r}}(t)$ that are shifted with respect to the point $\tau=0$ by -1 and +1 , respectively. This statement is illustrated well in Figs. 2-4. Figure 2 demonstrates the autocorrelation function $K(\tau)$ of some reference signal $f_{\mathrm{r}}(t)$.

Figure 3 illustrates the process of obtaining $D(\tau)$ in the form of the difference of the autocorrelation functions $K(\tau-1)$ and $K(\tau+1)$.

The method of stabilization of the steepness of the slope of the DC $D(\tau)[6,9]$ consists in dividing the function $D(\tau)$ at some point $\tau$ by the value of its derivative $D^{\prime}(\tau)$ at the point $\tau=0$. Taking into account that, in the general case, the function $D(\tau)$ is not symmetric with respect to the origin, one should calculate two values of the derivative at the point $\tau=0$ : one for a positive increment of the argument $\tau$, and the other, for a negative $\tau: K^{+}=D(-1)-D(0)$ and $K^{-}=D(+1)-$ $D(0)$. Since $D(0) \equiv 0$, we have $K^{+}=D(-1)$ and $K^{-}=$ $D(1)$, and the formulas for the normalization coefficients are expressed as follows:

$$
\begin{aligned}
K^{+} & =\sum_{t=0}^{L-1}\left[f_{\mathrm{r}}(t-1)-f_{\mathrm{r}}(t+1)\right] f_{\mathrm{r}}(t-1), \\
K^{-} & =\sum_{t=0}^{L-1}\left[f_{\mathrm{r}}(t-1)-f_{\mathrm{r}}(t+1)\right] f_{\mathrm{r}}(t+1) .
\end{aligned}
$$

Hence, if $D(\tau)>0$ for a current signal $f_{\mathrm{c}}(t)$, the value of the stabilized $D C$ is $S D(\tau)=D(\tau) / K^{+}$; otherwise $S D(\tau)=D(\tau) / K^{-}$.

The main drawbacks of the classical method of constructing a DC by formula (5) are the following:

- the impossibility of constructing a DC with wide MPWR and MWR;

- the problem of stabilization of a DC over the whole interval of definition of the MWR.

To eliminate these drawbacks, we modify our approach.

The main distinctive features of the proposed method for constructing a DC are as follows.

1. When calculating the current value of the DC, we propose using, instead of $f_{\mathrm{r}}(t)$ and $f_{\mathrm{c}}(t)$ of length $L$,

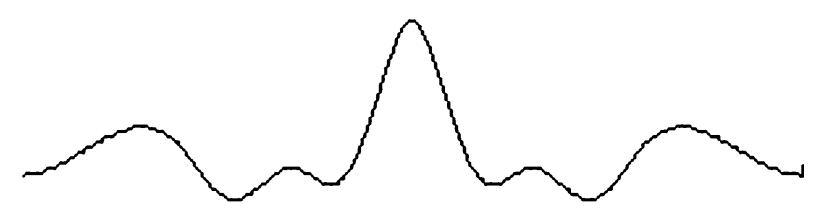

Fig. 2. Autocorrelation function $K(\tau)$.

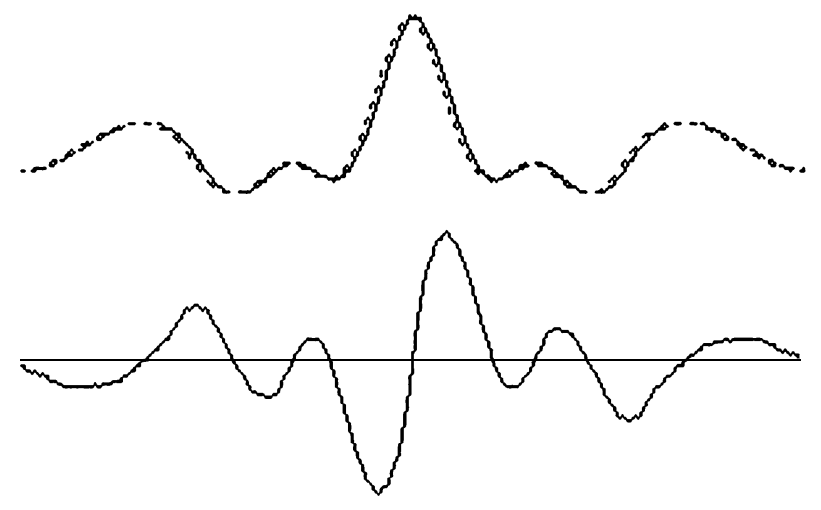

Fig. 3. $D(\tau)$ as difference of autocorrelation functions $K(\tau-1)$ and $K(\tau+1)$.

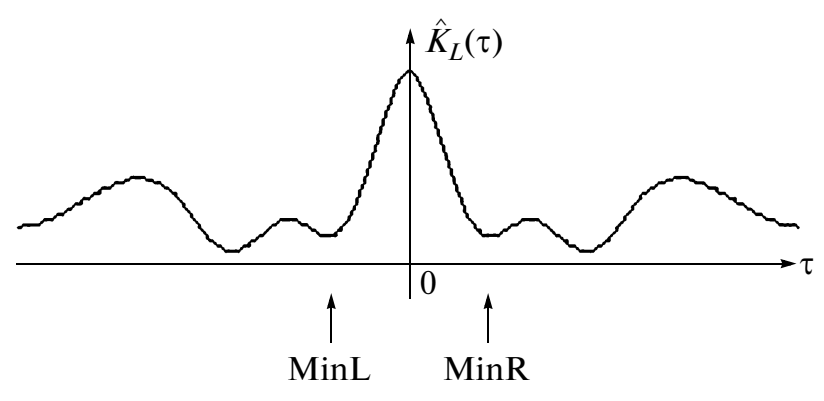

Fig. 4. Autocorrelation function $\hat{K}_{L}(\tau)$ of signal $f_{\mathrm{r}}(t)$.

signals $\hat{f}_{\mathrm{r}}(t)$ and $\hat{f}_{\mathrm{c}}(t)$ of reduced length $L_{3}=L-2 \theta$, which are related to $f_{\mathrm{r}}(t)$ and $f_{\mathrm{c}}(t)$ by the formulas

$$
\begin{gathered}
\hat{f}_{\mathrm{r}}(t)=f_{\mathrm{r}}(t+\theta), \quad \hat{f}_{\mathrm{c}}(t)=f_{\mathrm{c}}(t+\theta), \\
t=\overline{0-\left(L_{3}-1\right)},
\end{gathered}
$$

where $\theta$ is a parameter that defines the width of the MWR.

2. The value of the DC $D(\tau)$ should be calculated as the difference of cyclic autocorrelation functions of the reference signal $\hat{f}_{\mathrm{r}}(t)$ that have been separated by $\theta$ with respect to point $\tau=0$. For this purpose, one should apply, instead of the derivative $f_{\mathrm{r}}^{\prime}(t)$ of reference signal (3), the difference of two functions $\hat{f}_{\mathrm{r}}^{R}(t)$ 


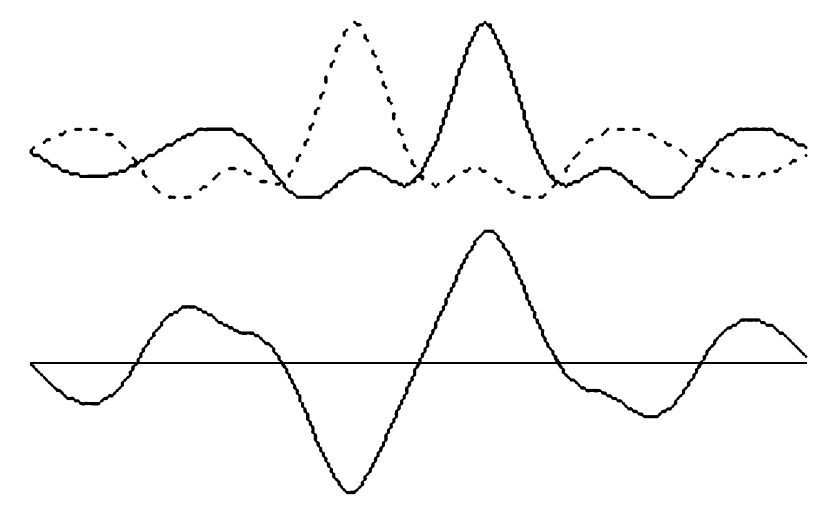

Fig. 5. Construction of function $D_{\theta}(\tau)$.

and $\hat{f}_{\mathrm{r}}^{L}(t), \hat{f}_{\mathrm{r}}^{\prime}(t)=\hat{f}_{\mathrm{r}}^{R}(t)-\hat{f}_{\mathrm{r}}^{L}(t)$ of length $L_{3}$, which are related to the reference signal $\hat{f}_{\mathrm{r}}(t)$ by the formulas

$$
\hat{f}_{\mathrm{r}}^{R}(t)=\hat{f}_{\mathrm{r}}(t \ominus \theta), \quad \hat{f}_{\mathrm{r}}^{L}(t)=\hat{f}_{\mathrm{r}}(t \oplus \theta),
$$

where $\ominus$ and $\oplus$ are the operations of subtraction and addition $\bmod L_{3}$, respectively. Then the value of $D_{\theta}(\tau)$ is calculated as follows:

$$
D_{\theta}(\tau)=\sum_{t=0}^{L_{\mathrm{r}}-1}\left[\hat{f}_{\mathrm{r}}^{R}(t)-\hat{f}_{\mathrm{r}}^{L}(t)\right] \hat{f}_{\mathrm{c}}(t) .
$$

Opening the brackets and making necessary substitutions, we finally obtain

$$
\begin{gathered}
D_{\theta}(\tau)=\sum_{t=0}^{L_{\mathrm{r}}-1}\left[\hat{f}_{\mathrm{r}}^{R}(t)-\hat{f}_{\mathrm{r}}^{L}(t)\right] \hat{f}_{\mathrm{c}}(t) \\
=\sum_{t=0}^{L_{\mathrm{r}}-1}\left[\hat{f}_{\mathrm{r}}(t \ominus \theta)-\hat{f}_{\mathrm{r}}(t \oplus \theta)\right] f_{\mathrm{r}}(t-\tau) \\
=\sum_{t=0}^{L_{\mathrm{r}}-1} \hat{f}_{\mathrm{r}}(t \ominus \theta) f_{\mathrm{r}}(t-\tau)-\sum_{t=0}^{L_{\mathrm{r}}-1} \hat{f}_{\mathrm{r}}(t \oplus \theta) f_{\mathrm{r}}(t-\tau) \\
=\hat{K}_{L_{3}}(t \ominus \theta)-\hat{K}_{L_{3}}(t \oplus \theta),
\end{gathered}
$$

where $\hat{K}_{L_{3}}(t \oplus \theta)$ and $\hat{K}_{L_{3}}(t \ominus \theta)$ are cyclic autocorrelation functions of the reference signal $\hat{f}_{\mathrm{r}}(t)$ of length $L_{3}$ that are cyclically shifted by $\pm \theta$ with respect to point $\tau=0$.

3. Reduction in the length of signals under processing to $L_{3}$ samples makes it possible to propose a new method for calculating coefficients $\mathrm{K}^{+}$and $\mathrm{K}^{-}$ to stabilize the steepness of $D_{\theta}(\tau)$, which eliminates the influence of edge effects on the DC within the MWR:

$$
\begin{gathered}
K_{\theta}=\sum_{t=0}^{L_{\mathrm{r}}-1}\left[\hat{f}_{\mathrm{r}}^{R}(t)-\hat{f}_{\mathrm{r}}^{L}(t)\right] \hat{f}_{\mathrm{r}}(t) ; \\
K_{\theta}^{+}=\sum_{t=0}^{L_{\mathrm{r}}-1}\left[\hat{f}_{\mathrm{r}}^{R}(t)-\hat{f}_{\mathrm{r}}^{L}(t)\right] \hat{f}_{\mathrm{r}}(t+2 \theta) .
\end{gathered}
$$

As a result, we obtain the following rule for calculating the value of the stabilized DC $S D_{\theta}(\tau)$. If $D_{\theta}(\tau)>$ 0 for the current signal $f_{\mathrm{c}}(t)$, then the value of the stabilized DC is

$$
S D_{\theta}(\tau)=D_{\theta}(\tau) \theta / K_{\theta}^{+}
$$

otherwise,

$$
S D_{\theta}(\tau)=D_{\theta}(\tau) \theta / K_{\theta} .
$$

Elimination of the influence of edge effects on the DC within the MWR is explained by the fact that, under, say, a left shift of $f_{\mathrm{c}}(t)$ by $\theta$ pixels, the signal $\hat{f}_{\mathrm{c}}(t)$ coincides with $f_{\mathrm{r}}(t)$; i.e., $\hat{f}_{\mathrm{c}}(t)=\hat{f}_{\mathrm{r}}(t+\theta), t=$ $\overline{0-\left(L_{3}-1\right)}$, and, accordingly, $D_{\theta}(\theta)=K_{\theta}$.

The algorithm for determining parameter $\theta$, which defines the width of the MWR, consists is as follows. At the preparatory stage, we calculate the cyclic autocorrelation function (Fig. 4)

$$
\hat{K}_{L}(\tau)=\sum_{t=0}^{L-1} f_{\mathrm{r}}(t) f_{\mathrm{r}}(t \oplus \tau)
$$

for the reference signal $f_{\mathrm{r}}(t), t=\overline{0-(L-1)}$.

To the left and right of the principal maximum of this function, we define the first two minima MinL and MinR, respectively.

The construction of function $D_{\theta}(\tau)$ is illustrated in Fig. 5, where functions $\hat{K}_{L}(\tau \oplus \theta)$ and $\hat{K}_{L}(\tau \ominus \theta)$ are shown by dotted and solid lines, respectively. This figure shows that the linear part of the MWR of function $D_{\theta}(\tau)$ lies in the interval $[-\theta, \theta]$ with the center at point $\tau=0$.

The maximum admissible value of parameter $\theta$ is sought by the following algorithm. For all $\theta, \theta=$ $\overline{0-\mathrm{MinR}}$, the mean absolute difference $E(\theta)$ is calculated between the values of the function $D_{\theta}(\tau)$ on the linear part of the MWR in the interval $[-\theta, \theta]$ and the inclined line described by the equation $\hat{D}_{\theta}(\tau)=\theta * \tau$, which is defined on the same interval. The maximum value of $\theta$ at which $E(\theta)$ does not exceed the prescribed error yields the sought value of the parameter $\theta$. 


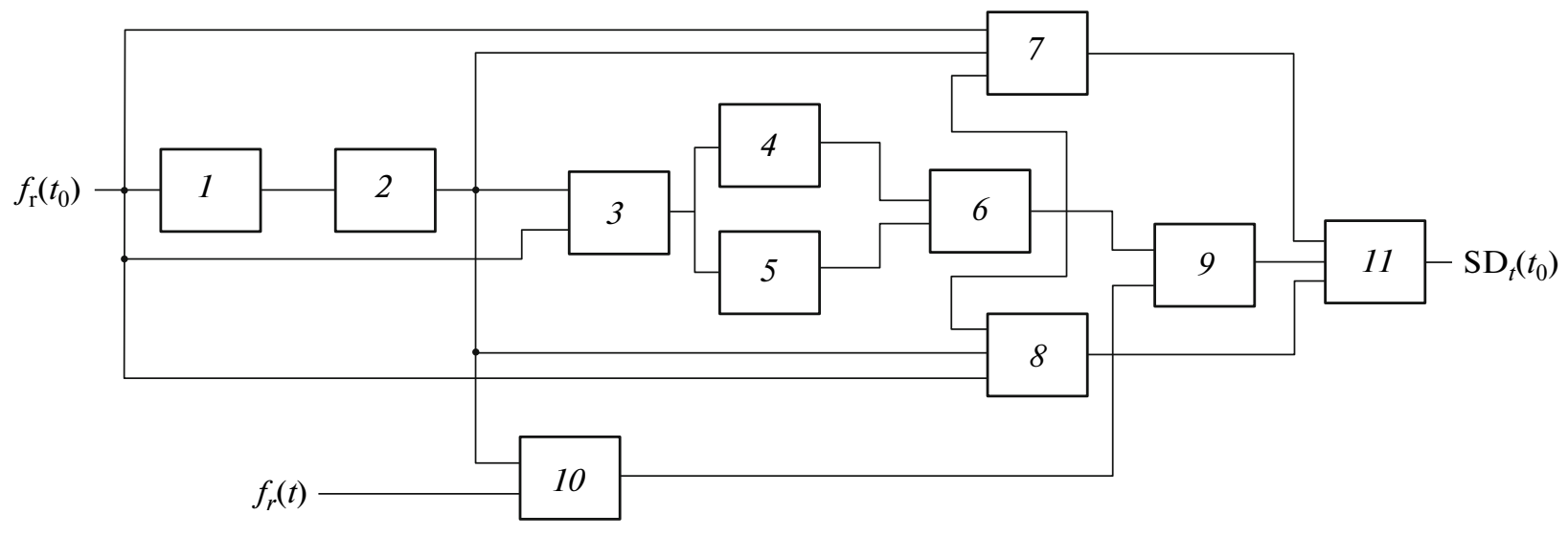

Fig. 6. Scheme of construction of modified CD.
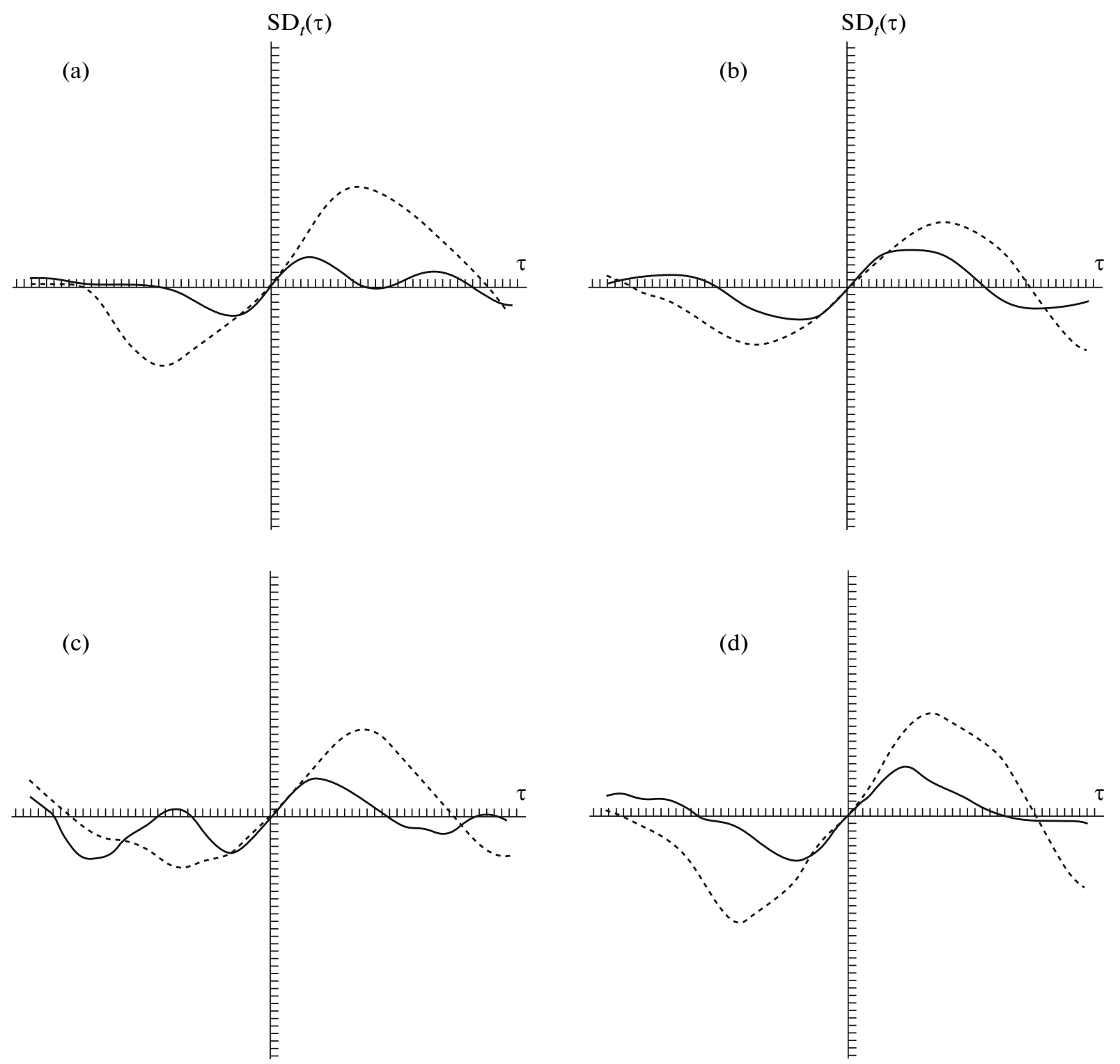

Fig. 7. Types of DCs of modified CD (solid line) and OCC CD (dotted line). 
Table 1

\begin{tabular}{|c|c|c|c|c|c|c|}
\hline No. & Mean OCC & Mean & $\frac{\text { Mean OCC }}{\text { Mean }}$ & Max OCC & Max & $\frac{\text { Max OCC }}{\operatorname{Max}}$ \\
\hline 1 & 1.964 & 0.971 & 2.02 & 8.165 & 1.626 & 5.02 \\
\hline 2 & 0.956 & 0.733 & 1.3 & 1.902 & 1.115 & 1.7 \\
\hline 3 & 0.645 & 0.881 & 0.73 & 3.173 & 1.451 & 2.19 \\
\hline 4 & 2.784 & 0.801 & 3.47 & 11.546 & 2.144 & 5.38 \\
\hline 5 & 1.887 & 0.99 & 1.9 & 12.003 & 1.7 & 7.05 \\
\hline 6 & 1.396 & 0.908 & 1.53 & 4.113 & 1.643 & 2.5 \\
\hline 7 & 0.7 & 0.934 & 0.749 & 2.046 & 1.596 & 1.28 \\
\hline 8 & 1.065 & 0.872 & 1.22 & 3.309 & 1.509 & 2.19 \\
\hline 9 & 2.171 & 0.902 & 2.4 & 10.133 & 2.55 & 3.96 \\
\hline 10 & 0.042 & 0.922 & 0.04 & 0.202 & 1.58 & 0.127 \\
\hline \multicolumn{3}{|c|}{ Mean } & 1.54 & Mean & & 3.14 \\
\hline
\end{tabular}

Table 2

\begin{tabular}{c|c|c|c|c|c|c|c}
\hline No. & Mean OCC & Mean & $\frac{\text { Mean OCC }}{\text { Mean }}$ & Max OCC & Max & $\frac{\text { Max OCC }}{\text { Max }}$ & Figure \\
\hline 1 & 0.069 & 0.005 & 13.8 & 0.214 & 0.017 & 12.6 & $\mathrm{a}$ \\
2 & 0.06 & 0.007 & 8.57 & 0.198 & 0.017 & 11.65 & $\mathrm{~b}$ \\
3 & 0.038 & 0.004 & 9.5 & 0.101 & 0.012 & 8.42 & $\mathrm{c}$ \\
4 & 0.023 & 0.005 & 4.6 & 0.057 & 0.013 & 4.38 & $\mathrm{~d}$ \\
\multicolumn{1}{l|}{ Mean } & & & 9.12 & Mean & & & 9.26 \\
\hline
\end{tabular}

Figure 6 shows the operation scheme of a CD that outputs a current value of the stabilized DC $S D_{\theta}(\tau)$. The whole operation process of the $C D$ is divided into two stages: the preparatory stage and the main stage. The preparatory stage is executed only once when forming the reference signal $f_{\mathrm{r}}(t)$ and is aimed at finding the following quantities: parameter $\theta$, difference $\hat{f}_{\mathrm{r}}^{\prime}(t)=\hat{f}_{\mathrm{r}}^{R}(t)-\hat{f}_{\mathrm{r}}^{L}(t)$, and normalization coefficients $K_{\theta}$ and $K_{\theta}^{+}$. This is done as follows. The reference signal is fed to the input of unit 1 , where the cyclic autocorrelation function $\hat{K}_{L}(\tau)$ of the signal $f_{\mathrm{r}}(t)$ is calculated. In unit 2, parameter $\theta$ is calculated on the basis of $\hat{K}_{L}(\tau)$ by the above-described algorithm. The value of parameter $\theta$ is transmitted to units $3,7,8$, and 10 . In unit 3 , the signal $\hat{f}_{\mathrm{r}}(t)=f_{\mathrm{r}}(t+$ $\theta), t=\overline{0-\left(L_{3}-1\right)}$ is formed. In units 4 and 5 , a cyclical shift of the signal $\hat{f}_{\mathrm{r}}(t)$ is performed to obtain $\hat{f}_{\mathrm{r}}^{R}(t)=\hat{f}_{\mathrm{r}}(t \ominus \theta)$ and $\hat{f}_{\mathrm{r}}^{L}(t)=\hat{f}_{\mathrm{r}}(t \oplus \theta)$, respectively. In unit 6, the difference $\hat{f}_{\mathrm{r}}^{\prime}(t)=\hat{f}_{\mathrm{r}}^{R}(t)-\hat{f}_{\mathrm{r}}^{L}(t)$ is calculated, which is then transmitted to units 7,8 , and 9. In units 7 and 8, the normalization coeffi- cients $K_{\theta}$ and $K_{\theta}^{+}$, respectively, are calculated on the basis of $f_{\mathrm{r}}(t)$, parameter $\theta$, and function $\hat{f}_{\mathrm{r}}^{\prime}(t), t=$ $\overline{0-\left(L_{3}-1\right)}$. This completes the preliminary stage. The main stage of operation starts with the arrival of the current signal $f_{\mathrm{c}}(t)$. In unit 10 , the function $\hat{f}_{\mathrm{c}}(t)=f_{\mathrm{c}}(t+\theta), t=\overline{0-\left(L_{3}-1\right)}$ is determined on the basis of $f_{\mathrm{c}}(t)$ and $\theta$ and transmitted to unit 9 to calculate the function $D_{\theta}\left(t_{0}\right)$ by formula (6). The final result is calculated in unit 11 on the basis of $D_{\theta}\left(t_{0}\right)$, $K_{\theta}^{-}$, and $K_{\theta}^{+}$in accordance with formulas (7) and (8).

\section{CONCLUSIONS}

To model the method for constructing a CD and compare it with an analog of the OCC CD [7, 8], we have constructed static DCs under conditions of the absence of noise. As a one-dimensional signal $f(t)$, $t=\overline{0-(L-1)}$, we constructed a function in the form of a sum of 50 normal distributions. To eliminate the sampling errors of the function $f^{k}(t)$ and to obtain, on its basis, a function $f_{\mathrm{c}}^{k}(t)$ with an arbitrary shift, we 

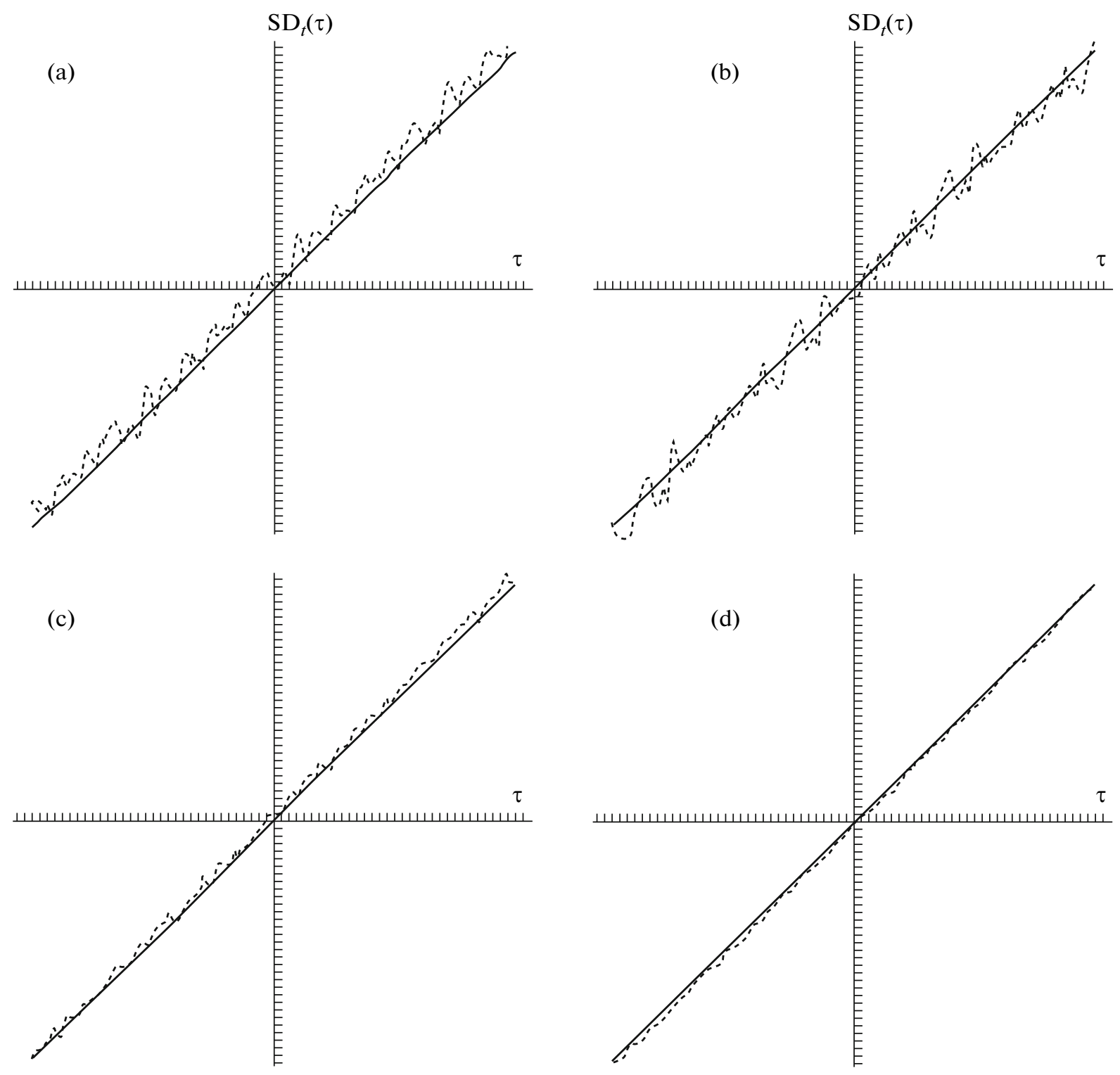

Fig. 8. Types of subpixel DCs of modified CD (solid line) and OCC CD (dotted line).

calculated the $f^{k}(t)$ value with regard to a minimum sampling increment of SubPixel $=0.005$ from the very beginning. Thus, $f^{k}(t)$ was constructed by the formula

$$
f^{k}(t)=\sum_{i=1}^{N} \exp \left(\frac{-\left(t-\operatorname{Pos}_{i}^{k}\right)^{2}}{10 *\left(\sigma_{i}^{k}+1\right)}\right),
$$

where $\kappa$ is the number of the realization of the function, $t=\overline{0-\left(L_{1} L_{2}-1\right)}, L_{1}=768, L_{2}=(1 /$ SubPixel $)$, and $N=50$.

The quantities $\operatorname{Pos}_{i}^{k}$ (shift) and $\sigma_{i}^{k}$ (scale) were formed randomly (by the RANDOM procedure) in the ranges [0-768] for $\operatorname{Pos}_{i}^{k}$ and [0-50] for $\sigma_{i}^{k}$.
The reference signal $f_{\mathrm{r}}^{K}(t)$ with length of $L=$ 300 pixels was formed from the central part of $f^{k}(t)$ by the formula $f_{\mathrm{r}}^{\mathrm{k}}(t)=f^{k}\left(\left(t+B_{\mathrm{r}}\right) * L_{2}\right), t=\overline{0-(L-1)}$, where $B_{\mathrm{r}}$ is the beginning of the reference signal, equal to $B_{\mathrm{r}}=(768 / 2-150)$.

The current signal $f_{\mathrm{c}}^{\mathrm{k}}(t)$ was formed from $f^{k}(t)$ by the formula $f_{\mathrm{c}}^{\mathrm{k}}(t)=f^{k}\left(\left(t+B_{\mathrm{r}}+\tau\right) * L_{2}\right), t=$ $\overline{0-(L-1)}$, where $\tau=\delta /$ SubPixel and $\delta$ is a shift that is a multiple of SubPixel.

We carried out 200 experiments in total. The step of the mutual shift of signals was one pixel. In total, we modeled \pm 160 shifts. Figure 7 demonstrates DCs of a modified CD (solid lines) and of an OCC CD (dotted 
lines). One division on the graphs corresponds to a shift of five pixels. The figures show that the MPWR for the OCC CD is nearly 1.5-2 times greater than that of the modified CD.

However, the modified CD has a lower error in determining the mutual shift of signals within the MWR than the OCC CD. Typical selected data for ten experiments are presented in Table 1 . One can see that the mean error of the OCC CD calculated within MWR in the interval $[-\theta, \theta]$ is greater than the error of the modified CD by a factor of 1.54 on average. A comparative analysis of the maximum error shows that the maximum error of the OCC CD is more than three times greater than that of the modified CD.

Figure 8 presents the types of subpixel DCs of the modified CD, which are shown by solid lines, and of the OCC CD, shown by dotted lines.

Simulation on estimating the subpixel mutual shifts of signals was carried out for \pm 160 shifts for a value of parameter $\theta=1$. The value of the shift was 0.005 pixels. The experimental data shown in Fig. 8 are summarized in Table 2.

\section{REFERENCES}

1. Yu. M. Astapov, D. V. Vasil'ev, and Yu. I. Zalozhnev, Theory of Optoelectronic Following-up Systems (Nauka, Moscow, 1988) [in Russian].

2. D. V. Vasil'ev, K. A. Grigor'ev, and V. A. Nikonov, "Role of boundary effects in invariant discriminator of image shift," Elektromagnit. Volny Elektron. Sist. 12 (9), 6171 (2007).

3. D. V. Vasil'ev, V. V. Sumerin, E. A. Firsov, and A. V. Gapon, "Direction autocapture according to image by means of sliding filtration," Elektromagnit. Volny Elektron. Sist. 12 (7), 49-52 (2007).
4. D. V. Vasil'ev and V. V. Mishin, "Invariant discriminators of image shift," Elektromagnit. Volny Elektron. Sist. 12 (7), 35-43 (2007).

5. E. A. Firsov, "Correlation astroorientator for spacecrafts," Elektromagnit. Volny Elektron. Sist. 12 (7), 44-48 (2007).

6. E. A. Firsov, "Development and application of correlation methods of image processing in high-speed optoelectronic follow-up systems," Extended Abstract of Candidate's Dissertation in Technical Sciences (Moscow Institute of Physics and Technology, Moscow, 2007).

7. A. V. Gapon, "Properties of image shift orthocorrelated discriminators," Elektromagnit. Volny Elektron. Sist. 14 (7), 23-29 (2009).

8. D. V. Vasil'ev and A. V. Gapon, "Elements of the theory for solving inverse correlation problems," Elektromagnit. Volny Elektron. Sist. 14 (7), 30-39 (2009).

9. V. N. Kruglov, "The way to stabilize gradient of discriminator curve slope," in Interuniversity Collection of Papers "Digital Methods in Control, Radiolocation and Communication (Ural State Univ., Sverdlovsk, 1986), pp. 119-126.

Translated by I. Nikitin

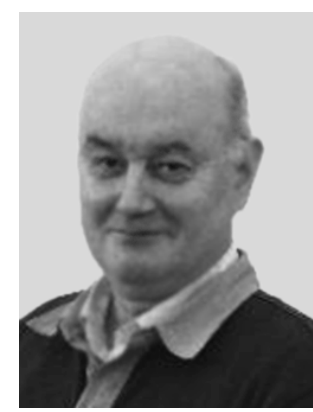

Vasilii N. Kruglov. Graduated from Radio Engineering Faculty, Ural Polytechnical Institute (UPI), in 1977. Received candidate's degree in engineering in 1989. Scientific interests: design, development, and application of machine vision systems in mining and engineering industries. 